\title{
Reacción de 18 líneas de arveja (Pisum sativum L.) a tres cepas de Fusarium oxysporum f. sp. pisi en Nariño, Colombia

\author{
Reaction of 18 climbing lines of pea (Pisum sativum L.) to three strains of \\ Fusarium oxysporum f. sp. pisi in Nariño, Colombia
}

\author{
Oscar Checa ${ }^{1 *}$, Francisco Alpala ${ }^{2}$ \\ Recibido para publicación: Mayo 18 de 2015 - Aceptado para publicación: Abril 20 de 2016
}

\begin{abstract}
RESUMEN
Uno de los problemas fitosanitarios limitantes del cultivo de arveja en Nariño, es el marchitamiento vascular causado por Fusarium oxysporum f. sp. pisi, que produce pérdidas entre el 30 y el $100 \%$ de la producción. El objetivo de esta investigación fue evaluar en condiciones de invernadero, la reacción de 18 genotipos de arveja volubles a tres aislamientos del hongo Fusarium oxysporum f. sp. pisi, procedentes de los municipios de Gualmatán, Ipiales y Pupiales ubicados en el departamento de Nariño. En lotes afectados, se realizó la colecta de muestras del tejido enfermo, a partir de plantas con síntomas de amarillamiento y presencia del hongo en la raíz, luego en laboratorio se obtuvieron cultivos monospóricos. La inoculación de los 18 genotipos de arveja se hizo con una concentración de $10^{6}$ conidias por mililitro. La evaluación se realizó utilizando un diseño irrestrictamente al azar con arreglo factorial combinatorio para dos factores. El factor A correspondió a los genotipos de arveja y el factor $\mathrm{B}$ a las tres procedencias del inóculo. Los aislamientos de $F$. oxysporum no afectaron la altura de las plantas hasta los 30 días después de la inoculación. Se destacaron las líneas UN7231-1, UN7143-3 y UN7328 por presentar reacción moderadamente resistente a Fusarium oxysporum f. sp. pisi para al menos dos de los tres aislamientos del patógeno. Se observó variabilidad genética en la reacción a Fusarium oxysporum f. sp. pisi dentro de las líneas evaluadas que ofrece la posibilidad de hacer selecciones individuales para generar líneas con resistencia más uniforme.
\end{abstract}

Palabras clave: genotipo, resistencia, inóculo, incidencia, escala, variabilidad, leguminosa, patógenos fungosos.

\begin{abstract}
Vascular wilt caused by Fusarium oxysporum f. sp. pisi is one of the phytosanitary problems limiting the pea crop in Nariño, which causes production losses between 30 and $100 \%$. The objective of this research was to evaluate, under greenhouse conditions, the reaction of 18 genotypes of climbing pea to three isolates of the fungus Fusarium oxysporum f. sp. pisi, from the municipalities of Gualmatán, Ipiales and Pupiales, located in the department of Nariño. In affected crops, the collection of samples of the diseased tissue was performed from plants with yellowing symptoms and presence of the fungus in the roots and then laboratory spore cultures were obtained. Inoculation of the 18 pea genotypes was made with a concentration of 106 conidia per milliliter. The evaluation was conducted using an unreservedly random factorial design with combinatorial arrangement to two factors. Factor A corresponded to the pea genotypesand factor $B$ to the threeinoculum sources. Isolates of $F$. oxysporum did not affect plantheight until 30 days after inoculation. The UN7231-1, UN7143-3 and UN7328 lines stood out for featuring a moderately resistant reaction to Fusarium oxysporum f. sp. pisi, for at least two of the three isolates of the pathogen. Within the evaluated lines, genetic variability in the reaction to Fusarium oxysporum f. sp. pisi was observed, whichoffers the possibility of making individual selections to create lines with a more uniform resistance.
\end{abstract}

Keywords: genotype, resistance, inoculum, incidence, scale, variability, legume, Pathogenic fungous.

\footnotetext{
1* Profesor Asociado Facultad de Ciencias Agrícolas Universidad de Nariño. Grupo de Investigación Cultivos Andinos GRICAND. e-mail cicagrarias@hotmail.com

${ }^{2}$ I.A. M.Sc. Grupo de Investigación Cultivos Andinos GRICAND Universidad de Nariño
} 


\section{INTRODUCCIÓN}

Uno de los problemas fitosanitarios limitantes de la zona andina de Nariño es el marchitamiento vascular causado por Fusarium oxysporum f. sp. pisi. Las pérdidas ocasionadas por este patógeno reducen del 30 al 100\% del rendimiento. Datos reportados en las zonas productoras de arveja del departamento de Nariño determinan que el $40 \%$ de la producción es afectada por este patógeno (Checa 1993). En los municipios de Pupiales, Ipiales y Gualmatán, considerados como los de mayor producción de arveja del Departamento de Nariño, se siembran las variedades ICACORPOICA Sindamanoy, San Isidro y Obonuco Andina, las cuales presentan susceptibilidad al marchitamiento ocasionado por $F$. oxysporum $\mathrm{f}$. sp. pisi, factores de influencia como el aumento del área sembrada y la implementación de los sistemas de tutorado que propician siembras repetidas en el mismo lote incrementaron la incidencia del patógeno (Quistial y Chávez 2009; Forero y Ligarreto 2009). En general el hongo produce una alteración típica y no del xilema con respecto a la translocación de agua, debido a la obstrucción del sistema radicular por la producción de masas de esporas, lo cual se observa en los marchitamientos vasculares (Checa 1993; Martínez et al. 2004).

En lotes afectados se observa parches con plantas amarillas, donde se aprecia un amarillamiento uniforme de la planta, causando la muerte en algunos casos o permaneciendo parcialmente viva en otros. Las plantas infectadas en estado de desarrollo más avanzados, comienzan con un amarillamiento en las hojas bajeras, que continua hacia las superiores, localizándose en el primero y segundo tercio de la planta, formando vainas muy pequeñas (Prieto 2008; Buitrago et al. 2006). Cuando se realiza la disección de la raíz, inicialmente, el micelio conforme madura adquiere una coloración que puede variar de crema a amarillo pálido. Bajo ciertas condiciones adquiere una tonalidad rosa pálido o algo púrpura, (Benavides y Muñoz 1998). En un examen más cuidadoso, se observan hojas enroscadas con márgenes caídos (Sañudo et al. 1999; Tamayo 2000; Kraft y Pfleger 2000). La resistencia genética, se constituye como una de las mejores opciones de control sin embargo, es una solución de difícil desarrollo, debido a la complejidad del medio en que se presenta la interacción patógenohospedero (Andrés et al. 1999; Madriz 2002 y Avendaño 2006).

El grupo de Cultivos Andinos de la Universidad de Nariño cuenta con una colección de trabajo de arveja con diferentes procedencias, compuesto por un juego de líneas que tienen como origen cruzamientos simples, dobles y múltiples. En algunos de ellos participaron líneas diferenciales de $F$. oxysporum propuestas por Haglund y Kraft (1979) para las diferentes razas del patógeno así como también las líneas Alemania 23 y Tolima 3 reportadas por Checa (1993). Estos genotipos se recibieron de la Universidad Nacional de Colombia, en generación F3 y luego fueron avanzados y uniformizados por selección masal hasta generación F6. De igual manera, se cuenta con genotipos procedentes del Banco de germoplasma de arveja de Corpoica.

Con 18 genotipos de arveja volubles seleccionados de la colección antes mencionada, se planteó el presente trabajo con el objeto de evaluar en condiciones de invernadero, su reacción a tres aislamientos del hongo Fusarium oxysporum f. sp. pisi, procedentes de las zonas productoras de arveja del departamento de Nariño ubicadas en los municipios de Gualmatán, Ipiales y Pupiales.

\section{MATERIALES Y MÉTODOS}

Esta investigación se desarrolló entre Octubre del 2011 y Julio 2013 en las instalaciones del Centro Internacional de Agricultura Limpia-Lope del SENA-Regional Nariño - 
municipio de Pasto, a una altura de 2630 msnm, zona agroecológica Fb (ICA, IGAC 1985); con temperatura promedia de $13{ }^{\circ} \mathrm{C}$, una precipitación de $803 \mathrm{~mm} / \mathrm{año}$ y humedad relativa del $82 \%$ (IDEAM 2005) en un suelo Vitric haplustands.

Material genético. Se utilizaron 18 líneas promisorias de arveja de la colección de trabajo del Grupo de Investigación en Cultivos Andinos (GRICAND), 15 de las cuales fueron aportadas inicialmente en generaciones $\mathrm{F} 3$ por el programa de mejoramiento de la Universidad Nacional y uniformizadas hasta F6 en la Universidad de Nariño, mediante selección masal por componentes de rendimiento. De igual manera se incluyeron tres genotipos con características deseables procedentes de Corpoica La Selva (Antioquia); como testigos comerciales se utilizó la variedad Andina. La reacción de los genotipos al patógeno se efectuó en invernadero. Se trabajó con tres aislamientos de F. oxysporum f. sp. pisi, procedentes de los municipios de Gualmatán, Ipiales y Pupiales; obtenidos y purificados en cajas Petri y medio PDA.

Muestras de tejido. La colecta de muestras del tejido enfermo, se realizó en lotes altamente afectados tomando tres plantas con síntomas de amarillamiento y presencia del hongo en la raíz. Se realizaron cortes de tejido afectado y se tomó pequeñas secciones de la parte interna del cuarto nudo del tallo, que fueron desinfectadas con hipoclorito de sodio al $1 \%$ por espacio de un minuto, luego se lavaron con agua destilada y se colocaron sobre toallas de papel absorbente estéril. Se sembraron cuatro trozos de tejido afectado en medio de cultivo (PDA). Después de la esporulación se realizó la identificación de acuerdo con las claves taxonómicas de Seifert (1996); Leslie y Summerell (2006).

Cultivos monospóricos. De cada uno de los aislamientos, se tomó un pequeño fragmento de medio de cultivo más hongo y se transfirió a un tubo de ensayo con $10 \mathrm{ml}$ de agua destilada estéril para luego agregarle dos gotas de dispersante Tween. Se agitó durante 5 minutos y después se tomaron $100 \mu$ l de la suspensión de conidias las cuales se esparcieron con la ayuda de un asa bacteriológica, sobre una caja Petri con medio de Cultivo PDA de capa delgada. Luego de ocho horas, de cada caja incubada se transfirió una espora germinada con la ayuda de una aguja insulínica a una nueva caja Petri con PDA (Fig. 1). A partir de los aislamientos monospóricos, se replicaron varias cajas Petri y se preparó el inóculo en una concentración de $1 \times 107$ conidias $/ \mathrm{ml}$, siguiendo las recomendaciones de Castellanos et al. (2011).
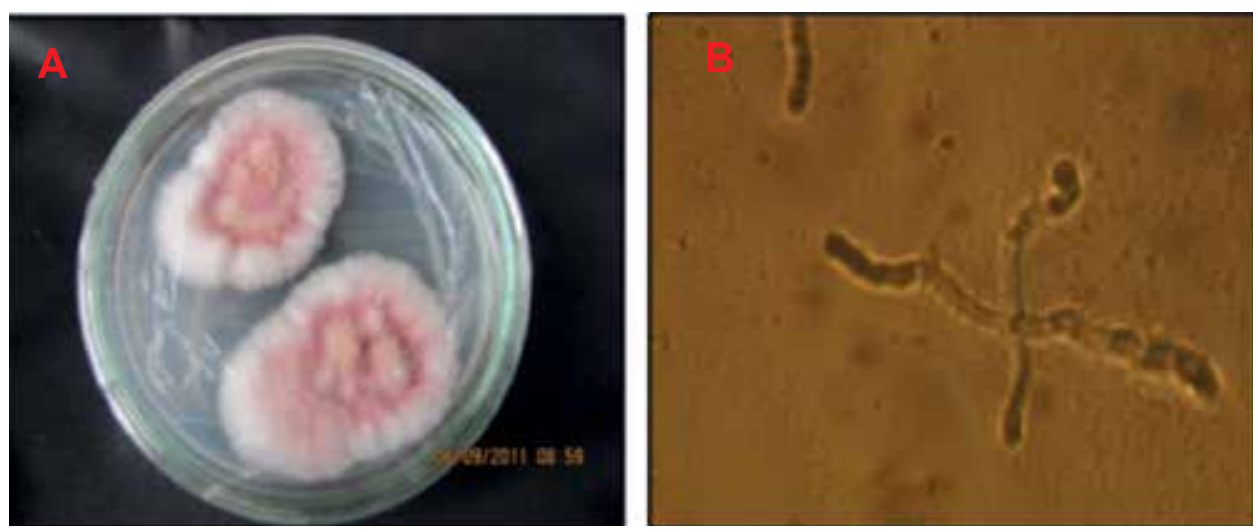

Figura 1: Cultivo monospórico de F. oxysporum. A. Aislamiento F. oxysporum f. sp. pisi Gualmatán.

B. Germinación de espora ocho horas después de sembrada. 
Inoculación y trasplante a materos. La semilla de los 18 genotipos se sembró en semilleros o bandejas plásticas con 50 alveolos. Cuando las plantas alcanzaron una altura de $8 \mathrm{~cm}$, fueron removidas de las bandejas, para lavar sus raíces con agua corriente, cortar sus extremos y luego sumergirlas en la suspensión de esporas por espacio de dos minutos. Finalmente, las plantas de cada línea se trasplantaron a materos que contenían un kilo de suelo de bosque natural, secado, y esterilizado de acuerdo con las recomendaciones de Ormeño y Zúñiga (1999).

Diseño experimental. Se utilizó un Diseño Irrestrictamente al Azar (DIA), con arreglo factorial combinatorio para dos factores y dos repeticiones. El factor A correspondió a los 18 genotipos más la variedad Andina que se usó como testigo comercial y como testigo absoluto (sin inoculación). El factor B lo constituyeron las tres procedencias del inoculo (Ipiales, Gualmatán y Pupiales). Por cada genotipo, procedencia de inoculo y repetición, se utilizaron cinco plantas o materos que correspondieron a la unidad experimental.

Variables evaluadas y análisis estadístico.

Altura de planta: Se realizaron tres lecturas a los 8, 15 y 30 días después de la inoculación, se midió la altura de cada una de las plantas en $\mathrm{cm}$., desde la base hasta el último foliolo; Incidencia de amarillamiento: se tomó el número de plantas con síntoma de amarillamiento registrando el resultado en porcentaje; decoloración de la raíz: se contó el número de plantas con presencia decoloración rojiza en la raíz observada mediante disección longitudinal de la misma y se llevó a porcentaje el resultado. En este punto se tomó una muestra de tejido para realizar el reaislamiento del hongo y confirmar la presencia de F. oxysporum. Las variables registradas en porcentaje, se transformaron mediante la fórmula arcoseno $\sqrt{ } \%$. Los resultados se sometieron a un análisis de varianza y prueba de comparación de promedios de Tukey. Por otra parte la reacción de los genotipos medida por amarillamiento y decoloración de raíz también se evaluó en una escala de 0 a 5 en donde $0=$ inmune, sin plantas afectadas; $1=$ resistente, con 1 a $20 \%$ de plantas afectadas; $2=$ moderadamente resistente con 21 a $40 \%$ de plantas afectadas; $3=$ moderadamente susceptibles con 41 a $60 \%$ de plantas afectadas; $4=$ susceptible con 61 a $80 \%$ de plantas afectadas; 5 = altamente susceptible con 81 a $100 \%$ de plantas afectadas.

\section{RESULTADOS Y DISCUSIÓN}

Las variables altura de planta a los 8 (ALT1), 15 (ALT2) y 30 (ALT3) días después de la inoculación, porcentaje de amarillamiento de plantas (AMIN\%) y porcentaje de plantas con decoloración de raíz (DRAIZ\%), presentaron diferencias significativas entre aislamientos, genotipos e interacción aislamiento $\mathrm{x}$ genotipo indicando que existió un comportamiento diferencial de los genotipos a través de los tres aislamientos (Ipiales, Gualmatán y Pupiales), en consecuencia la comparación de promedios de los genotipos se efectuó dentro de cada uno de los aislamientos, descartando la discusión de los efectos simples.

\section{Altura de planta}

Primera lectura (ALT1). Para el aislamiento de Gualmatán la altura de las plantas tomadas 8 días después del trasplante osciló entre 8,4 y $22 \mathrm{~cm}$. El $85 \%$ de las líneas no mostraron diferencias significativas. Las líneas UN7313 y UN7324 con $22 \mathrm{~cm}$ fueron significativamente más altas que las líneas ILS3597, ANDICON (Andina con inoculo) y ANDISIN (Andina sin inoculo) cuyos promedios estuvieron por debajo de 11,2 cm. Las líneas UN71432 y UN7103 con promedios de 17,66 y $18,6 \mathrm{~cm}$ respectivamente superaron al tratamiento Andina sin inoculación que alcanzó un promedio de 8,4 cm (Tabla 1).

Para el aislamiento de Ipiales, el $85 \%$ de las líneas no mostraron diferencias significativas entre sí, coincidiendo en porcentaje con el 
Tabla 1. Comparación de medias para la primera lectura de altura de plantas $(\mathrm{cm})$ a los ocho días después de la inoculación de 18 líneas y dos testigos de arveja voluble (Pisum sativum L.) con tres aislamientos de F. oxysporum f. sp. pisi.

\begin{tabular}{|c|c|c|c|c|c|c|c|c|}
\hline \multicolumn{3}{|c|}{ AISLAMIENTO GUALMATÁN } & \multicolumn{3}{|c|}{ AISLAMIENTO IPIALES } & \multicolumn{3}{|c|}{ AISLAMIENTO PUPIALES } \\
\hline \multirow{2}{*}{$\begin{array}{l}\text { GENOTIPO } \\
\text { UN7324 }\end{array}$} & \multicolumn{2}{|c|}{ PROMEDIO } & \multirow{2}{*}{$\begin{array}{l}\text { GENOTIPO } \\
\text { UN7325 }\end{array}$} & \multicolumn{2}{|c|}{ PROMEDIO } & \multirow{2}{*}{$\begin{array}{l}\text { GENOTIPO } \\
\text { UN7325 }\end{array}$} & \multicolumn{2}{|c|}{ PROMEDIO } \\
\hline & 22 & a & & 22,32 & a & & 22,39 & a \\
\hline UN7313 & 21,23 & a & UN7313 & 21,07 & $a b$ & UN7313 & 22,11 & a \\
\hline UN7103 & 18,6 & $a b$ & UN7143-2 & 16,94 & $a b c$ & UN7115 & 19,39 & $\mathrm{ab}$ \\
\hline UN7143-2 & 17,66 & $a b$ & UN7103 & 16,85 & $a b c$ & ANDICON & 19,3 & $a b$ \\
\hline ILS3621 & 17,12 & $a b c$ & UN7364 & 16,15 & abcd & ILS3593 & 18,8 & $\mathrm{ab}$ \\
\hline UN7370-1 & 16,91 & $a b c$ & UN7324 & 16,07 & abcd & UN7103 & 18,53 & $a b$ \\
\hline UN7328 & 16,87 & $a b c$ & ANDICON & 15,52 & abcd & UN7364 & 17,47 & $\mathrm{ab}$ \\
\hline ILS3593 & 16,57 & $a b c$ & UN7115 & 14,94 & abcd & UN7328 & 16,98 & $a b$ \\
\hline UN7143-3 & 16,28 & $a b c$ & UN7371-2 & 14,89 & abcd & UN7371-2 & 16,65 & $a b$ \\
\hline UN7325 & 16,04 & $a b c$ & UN7232-1 & 14,82 & abcd & UN7336 & 16,18 & $a b$ \\
\hline UN7143-1 & 15,07 & $a b c$ & UN7143-1 & 14,21 & abcd & UN7143-3 & 16,17 & $a b$ \\
\hline UN7336 & 14,92 & $a b c$ & ILS3597 & 13,75 & abcd & UN7370-1 & 15,26 & $a b$ \\
\hline UN7115 & 14,7 & $a b c$ & UN7143-3 & 13,61 & abcd & UN7143-2 & 14,61 & $a b$ \\
\hline UN7364 & 14,7 & $a b c$ & UN7328 & 12,87 & bcd & UN7100 & 13,98 & $a b$ \\
\hline UN7232-1 & 14,43 & $a b c$ & UN7100 & 12,27 & bcd & UN7332-1 & 13,94 & $a b$ \\
\hline UN7371-2 & 13,7 & $a b c$ & UN7336 & 12,26 & bcd & UN7324 & 13,43 & $a b$ \\
\hline UN7100 & 13,6 & $a b c$ & ANDISIN & 11,92 & bcd & ILS3621 & 12,7 & $\mathrm{~b}$ \\
\hline ILS3597 & 11,16 & $\mathrm{bc}$ & ILS3621 & 11,06 & $\mathrm{~cd}$ & ILS3597 & 12,42 & b \\
\hline ANDICON & 9,7 & bc & UN7370-1 & 10,25 & $\mathrm{~cd}$ & UN7143-1 & 11,94 & b \\
\hline ANDISIN & 8,4 & c & ILS3593 & 7,5 & d & ANDISIN & 11,27 & b \\
\hline Prom. Loc. & 15,48 & & & 14,463 & & & 16,176 & \\
\hline Comp. Tukey & 9,21 & & & & & & & \\
\hline
\end{tabular}

Valores con diferente letra son significativos al nivel del 5\%. Prueba de Tuckey.

encontrado en el de Gualmatán, no obstante en el aislamiento de Ipiales se destacó la línea UN7325 con 22,32 cm, presentando una altura significativamente mayor que las líneas UN7328, UN7100, UN7336, Andina sin inoculo, ILS3621, UN7370-1 e ILS3593, que estuvieron entre $12,87 \mathrm{~cm}$ y $7,5 \mathrm{~cm}$. De igual manera sobresale la línea UN7313 con 21,07 $\mathrm{cm}$ con diferencias significativas respecto a las líneas ILS3621, UN7370-1 e ILS3597 que presentaron menos de 11,1 cm (Tabla 1). En el aislamiento de Pupiales, el $90 \%$ de líneas mostraron igualdad estadística en la altura de la planta para esta lectura, sin embargo las líneas UN7325 y UN7313 sobresalen presentando promedios de 22,39 y 22,11 cm significativamente más altos respecto a ILS3621, ILS3597, UN7143-1 y ANDISIN (Andina sin inoculo) que estuvieron por debajo de $12,8 \mathrm{~cm}$ (Tabla 1).

En los tres aislamientos, no se observó diferencias significativas entre los testigos Andina con y sin inoculación, indicando que a los ocho días después de la inoculación el hongo no afectó la altura de las plantas y las diferencias observadas entre los genotipos pueden atribuirse a los genes que controlan la expresión de este carácter.

Segunda lectura (ALT2). Para el aislamiento de F. oxysporum procedente de Gualmatán, la altura de las plantas tomadas 15 días después del trasplante osciló entre 16,41 y 29,65 cm. El $85 \%$ de las líneas no mostraron diferencias significativas, sin embargo la línea UN7324 con 
29,65 cm fue significativamente más alta que UN7143-3, ANDICON (Andina con inoculo) y ANDISIN (Andina sin inoculo) cuyos promedios estuvieron por debajo de $17 \mathrm{~cm}$ - Por otra parte la línea UN7313 con 29,22 cm superó al testigo ANDISIN (Andina sin inoculo) que alcanzó un promedio de 16,75 cm (Tabla 2).

Respecto al aislamiento de Ipiales se observó que el $80 \%$ de las líneas no mostraron diferencias estadísticas significativas entre sí, no obstante, sobresalen las líneas UN7313 y UN7325 con 26,22 y $29,81 \mathrm{~cm}$, presentando una altura significativamente mayor que las líneas ILS3593, ILS3621, ILS3597, UN7370-1 y ANDISIN (Andina sin inoculo) cuyo promedio varió entre 13,56 y 17,22 cm (Tabla 2).
Para el aislamiento de $F$. oxysporum de Pupiales, el $95 \%$ de las líneas mostraron igualdad estadística en la altura de la planta, sin embargo UN7325 con 30,79 cm supera significativamente a las líneas ILS3597 y UN7100 que presentaron 17,67 y $18,18 \mathrm{~cm}$ respectivamente (Tabla 2 ). En esta lectura de altura (ALT2), la línea UN7325 se destacó para los aislamientos de Ipiales y Pupiales, alcanzando promedios de 29,81 y $30,79 \mathrm{~cm}$ y en comparación con la lectura tomada ocho días después de la inoculación, mantuvo su tendencia.

La comparación entre los testigos Andina con inoculación y Andina sin inoculación, no mostró diferencias significativas, conservando la misma tendencia observada en la primera

Tabla 2. Comparación de medias para la segunda lectura de altura de plantas ( $\mathrm{cm}$ ) a los quince días después de la inoculación de 18 líneas y dos testigos de arveja voluble (Pisum sativum L.) con tres aislamientos de F. oxysporum f. sp. pisi.

\begin{tabular}{lllllllll}
\hline \multicolumn{2}{c}{ AISLAMIENTO GUALMATÁN } & \multicolumn{2}{c}{ AISLAMIENTO IPIALES } & \multicolumn{3}{c}{ AISLAMIENTO PUPIALES } \\
GENOTIPO & PROMEDIO & GENOTIPO & PROMEDIO & GENOTIPO & PROMEDIO \\
\hline UN7324 & 29,65 & $\mathrm{a}$ & UN7325 & 29,81 & $\mathrm{a}$ & UN7325 & 30,79 & $\mathrm{a}$ \\
UN7313 & 29,22 & $\mathrm{ab}$ & UN7313 & 26,22 & $\mathrm{a}$ & ILS3593 & 29,33 & $\mathrm{ab}$ \\
UN7364 & 28,49 & $\mathrm{abc}$ & UN7103 & 22,92 & $\mathrm{ab}$ & UN7313 & 27,26 & $\mathrm{ab}$ \\
UN7103 & 24,71 & $\mathrm{abc}$ & UN7324 & 22,88 & $\mathrm{ab}$ & UN7115 & 24,82 & $\mathrm{ab}$ \\
ILS3621 & 23,97 & $\mathrm{abc}$ & UN7143-2 & 22,21 & $\mathrm{ab}$ & UN7143-3 & 23,75 & $\mathrm{ab}$ \\
UN7370-1 & 23,78 & $\mathrm{abc}$ & UN7115 & 21,88 & $\mathrm{ab}$ & UN7364 & 23,65 & $\mathrm{ab}$ \\
ILS3593 & 23,7 & $\mathrm{abc}$ & UN7232-1 & 21,23 & $\mathrm{ab}$ & ANDICON & 23,13 & $\mathrm{ab}$ \\
UN7325 & 23,34 & $\mathrm{abc}$ & UN7328 & 20,65 & $\mathrm{ab}$ & UN7328 & 22,61 & $\mathrm{ab}$ \\
ILS3597 & 22,89 & $\mathrm{abc}$ & UN7364 & 19,69 & $\mathrm{ab}$ & UN7371-2 & 22,29 & $\mathrm{ab}$ \\
UN7115 & 22,56 & $\mathrm{abc}$ & UN7143-1 & 18,99 & $\mathrm{ab}$ & UN7232-1 & 21,94 & $\mathrm{ab}$ \\
UN7143-2 & 22,55 & $\mathrm{abc}$ & UN7371-2 & 18,98 & $\mathrm{ab}$ & UN7103 & 21,7 & $\mathrm{ab}$ \\
UN7328 & 22,26 & $\mathrm{abc}$ & UN7100 & 18,78 & $\mathrm{ab}$ & UN7370-1 & 21,51 & $\mathrm{ab}$ \\
UN7100 & 20,16 & $\mathrm{abc}$ & ANDICON & 17,97 & $\mathrm{ab}$ & ANDISIN & 20,3 & $\mathrm{ab}$ \\
UN7143-1 & 20,15 & $\mathrm{abc}$ & UN7143-3 & 17,81 & $\mathrm{ab}$ & UN7143-1 & 20,23 & $\mathrm{ab}$ \\
UN7232-1 & 19,67 & $\mathrm{abc}$ & UN7336 & 17,24 & $\mathrm{ab}$ & UN7143-2 & 20,19 & $\mathrm{ab}$ \\
UN7371-2 & 19,37 & $\mathrm{abc}$ & ANDISIN & 17,22 & $\mathrm{~b}$ & UN7336 & 20,11 & $\mathrm{ab}$ \\
UN7336 & 19,23 & $\mathrm{abc}$ & UN7370-1 & 16,62 & $\mathrm{~b}$ & ILS3621 & 19,1 & $\mathrm{ab}$ \\
UN7143-3 & 16,95 & $\mathrm{bc}$ & ILS3597 & 15,3 & $\mathrm{~b}$ & UN7324 & 18,28 & $\mathrm{ab}$ \\
ANDISIN & 16,75 & $\mathrm{bc}$ & ILS3621 & 15,08 & $\mathrm{~b}$ & UN7100 & 18,18 & $\mathrm{~b}$ \\
ANDICON & 16,41 & $\mathrm{c}$ & ILS3593 & 13,56 & $\mathrm{~b}$ & ILS3597 & 17,67 & $\mathrm{~b}$ \\
Prom. Loc. & 22,29 & & & 19,75 & & & 22,34 & \\
Comp. Tukey & 12,57 & & & & & & & \\
\hline ValOres c0 & & & & & & &
\end{tabular}

Valores con diferente letra son significativos al nivel del 5\%. Prueba de Tuckey. 
lectura. Es posible que en el tratamiento Andina con inoculación, el patógeno, haya ingresado al testigo susceptible, pero su proceso de colonización no sea tan agresivo como para afectar de manera significativa la altura de las plantas, por lo tanto al igual que en la primera lectura, las diferencias entre los genotipos en la segunda lectura se puede atribuir a la variabilidad en los genes que condicionan este rasgo. Según Muñoz (2012), en la expresión del carácter altura de plantas, están involucrados uno o pocos genes mayores, con poca interacción con el ambiente y genes menores de herencia cuantitativa que son altamente afectados por el ambiente.

Tercera lectura (ALT3). Los resultados de la lectura realizada a los 30 días después de la inoculación, indican que en el aislamiento de Gualmatán el 95\% de los genotipos evaluados no presentaron diferencia significativas entre sí, incluido en este grupo los testigos Andina con y Andina sin inoculación, confirmando los resultados obtenidos en las lecturas realizadas a los 8 y 15 días después de la inoculación (ALT1 y ALT2), no obstante nueve líneas con alturas entre 41,42 y 48,54 cm, mostraron diferencias a nivel del $5 \%$ de probabilidad con respecto al tratamiento ANDISIN (Andina sin inoculación) que alcanzó un promedio de 18,9 cm (Tabla 3).

Para el aislamiento de Ipiales la altura de las líneas evaluadas a los 30 días después de la inoculación, varió entre 26,17cm y 46,5cm sin diferencias significativas entre los tratamientos evaluados (Tabla 3). Finalmente, para el aislamiento de Pupiales la altura osciló entre 24,78 y 51,56 cm, observándose uniformidad en el $85 \%$ de los genotipos. Las líneas UN7103, ILS3593 y UN7325, con promedios entre 51,65 y 48,56 mostraron diferencias significativas respecto a ILS3621, ISL3597, UN7143-1 que alcanzaron entre 26,27 y $24,78 \mathrm{~cm}$. En ambos aislamientos los testigos Andina con y sin inoculación, no presentaron diferencias estadísticas (Tabla 3).
En general, al analizar la altura de planta en las tres lecturas realizadas, se observó alta uniformidad entre las líneas evaluadas. Las pocas diferencias están más relacionadas con los genes que responden por la característica de la altura de planta, que con los genes de resistencia o susceptibilidad al patógeno. Lo anterior se confirma al observar resultados similares en los tratamientos ANDICON (Andina con Inoculación) y ANDISIN (Andina sin inoculación). Es posible que los efectos del patógeno sobre la altura de la planta sean observables en evaluaciones posteriores a los 30 días de la inoculación. Al respecto Rodríguez (2006), encontró que los efectos de $F$. oxysporum sobre la altura de la planta no fueron evidentes a los 30 días después del trasplante, pero si lo fueron a los 60 días. Kraft y Haglund (1978), argumentan que, solamente a partir de los 30 días después de la inoculación, el 30\% de las plantas presentó síntomas externos de la enfermedad generando algunas dudas en la calificación al momento de realizar la observación. Además, el número de plantas con síntomas alcanzó un máximo a los 60 días después de la inoculación. Esta situación no es deseable en la investigación, dado que en campo las plantas de la variedad susceptible afectadas por $F$. oxysporum f. sp. pisi en forma natural, expresan síntomas claros de la enfermedad entre los 20 y 30 días después de la siembra. En contraste Calderón et al. (2000), afirman que es en la época de floración en donde se manifiesta el mayor número de plantas con síntomas, lo cual se atribuye al cambio de la etapa vegetativa a la reproductiva que implica un gasto adicional de energía, circunstancia que aprovecha el hongo para desarrollarse y afectar el crecimiento de la planta.

Incidencia de amarillamiento (AMIN). La evaluación realizada con el aislamiento de Gualmatán, mostró que los porcentajes de amarillamiento estuvieron entre 10,18 y $57,1 \%$. El $80 \%$ de los tratamientos evaluados 
Tabla 3. Alturas en invernadero Treinta días después de inocular 18 líneas y dos testigos de arveja voluble (Pisum sativum L.) con tres aislamientos de Fusarium oxysporum f. sp. pisi

\begin{tabular}{|c|c|c|c|c|c|c|c|c|}
\hline \multicolumn{3}{|c|}{ AISLAMIENTO GUALMATÁN } & \multicolumn{3}{|c|}{ AISLAMIENTO IPIALES } & \multicolumn{3}{|c|}{ AISLAMIENTO PUPIALES } \\
\hline \multirow{2}{*}{$\frac{\text { GENOTIPO }}{\text { ILS3621 }}$} & \multicolumn{2}{|c|}{ PROMEDIO } & \multirow{2}{*}{$\begin{array}{l}\text { GENOTIPO } \\
\text { UN7325 }\end{array}$} & \multicolumn{2}{|c|}{ PROMEDIO } & \multirow{2}{*}{$\begin{array}{l}\text { GENOTIPO } \\
\text { UN7103 }\end{array}$} & \multicolumn{2}{|c|}{ PROMEDIO } \\
\hline & 48,54 & $\mathrm{a}$ & & 46,5 & $\mathrm{a}$ & & 51,65 & a \\
\hline UN7324 & 47,83 & $\mathrm{a}$ & UN7364 & 45,51 & $\mathrm{a}$ & ILS3593 & 51,02 & $a b$ \\
\hline ILS3593 & 45,18 & $\mathrm{a}$ & UN7313 & 39,49 & $\mathrm{a}$ & UN7325 & 48,56 & $a b$ \\
\hline UN7370-1 & 44,29 & $\mathrm{a}$ & UN7103 & 39,01 & a & UN7115 & 42,09 & $a b c$ \\
\hline UN7313 & 43,32 & $\mathrm{a}$ & UN7324 & 37,37 & $\mathrm{a}$ & UN7313 & 40,49 & $a b c$ \\
\hline UN7328 & 41,78 & a & ILS3593 & 37,02 & $\mathrm{a}$ & UN7143-3 & 39.49 & $a b c$ \\
\hline UN7115 & 41,73 & $\mathrm{a}$ & UN7232-1 & 36,94 & $\mathrm{a}$ & UN7364 & 38,11 & $a b c$ \\
\hline UN7103 & 41,73 & $\mathrm{a}$ & UN7336 & 36,35 & $\mathrm{a}$ & UN7336 & 37,14 & $a b c$ \\
\hline UN7336 & 41,42 & $\mathrm{a}$ & ANDICON & 36,17 & $\mathrm{a}$ & UN7371-2 & 35,2 & $a b c$ \\
\hline UN7325 & 39,69 & $a b$ & UN7143-2 & 36,08 & a & UN7328 & 34,63 & $a b c$ \\
\hline UN7143-2 & 39,5 & $a b$ & UN7115 & 35,91 & $\mathrm{a}$ & UN7324 & 34,23 & $a b c$ \\
\hline UN7100 & 38,78 & $\mathrm{ab}$ & ANDISIN & 35,3 & a & UN7370-1 & 33,73 & $\mathrm{abc}$ \\
\hline UN7143-3 & 37,42 & $a b$ & UN7370-1 & 33,86 & $\mathrm{a}$ & UN7232-1 & 32,84 & $a b c$ \\
\hline UN7143-1 & 37,29 & $a b$ & UN7143-1 & 33,59 & $\mathrm{a}$ & ANDISIN & 31,63 & $a b c$ \\
\hline UN7232-1 & 35,42 & $a b$ & UN7328 & 33,03 & a & UN7143-2 & 30,01 & $a b c$ \\
\hline UN7364 & 34,2 & $\mathrm{ab}$ & UN7100 & 32,47 & a & ANDICON & 29,66 & bc \\
\hline ILS3597 & 31,86 & $a b$ & UN7371-2 & 32,41 & $\mathrm{a}$ & UN7100 & 29,42 & bc \\
\hline UN7371-2 & 31,41 & $a b$ & ILS3621 & 30,41 & $\mathrm{a}$ & ILS3621 & 26,27 & c \\
\hline ANDICON & 28,51 & $a b$ & UN7143-3 & 28,31 & $\mathrm{a}$ & ILS3597 & 25,37 & c \\
\hline ANDISIN & 18,95 & b & ILS3597 & 26,17 & $\mathrm{a}$ & UN7143-1 & 24,78 & c \\
\hline Prom. Loc. & 38,44 & & & 35,60 & & & 33,84 & \\
\hline Comp. Tukey & 21,67 & & & & & & & \\
\hline
\end{tabular}

Valores con diferente letra son significativos al nivel del 5\%. Prueba de Tuckey.

no presentaron diferencias significativas entre sí. Los tratamientos ILS3621, UN7370-1 y ANDISIN con promedios de 10,18\%, mostraron incidencia significativamente menor que la presentada por las líneas UN7103, UN71432, UN7100, ILS3597, UN7364, UN7143-3 y UN7324, que osciló entre 57,1 y 45\%. (Tabla 4).

Para el aislamiento de Ipiales se encontró porcentajes de incidencia entre 10,18 y $44,99 \%$ con poca variación entre los tratamiento evaluados. Las líneas UN7232-1, UN71432, UN7115, ILS3597 y el testigo ANDISIN (Andina sin inoculación) presentaron un bajo porcentaje con 10,18\% mostrando incidencia significativamente menor a la obtenida por UN7313 (44,99\%) (Tabla 4).

En el aislamiento de Pupiales se observaron mayores diferencias entre tratamientos. Los porcentajes de plantas con amarillamiento estuvieron entre 10,18 y 79,8\%. Los tratamientos UN7143-3, UN7370-1, ILS3593, UN7364 y ANDISIN (Andina sin inoculo) utilizado como testigo, mostraron la menor incidencia del patógeno con promedios entre 28,28 y $10,18 \%$, siendo significativamente menos afectadas respecto al testigo ANDICON (Andina con inoculación) y a las líneas UN7100, UN7143-1, ILS3621, UN7371-2 y UN7324 que tuvieron entre 79,8 y $65,28 \%$, de plantas con amarillamiento (Tabla 4).

Al hacer una análisis general del porcentaje de amarillamiento observado en los genotipos evaluados con los tres aislamientos de $F$. oxysporum, se observa que entre las líneas con menor incidencia aparece UN7370-1, sin embargo las otras líneas con baja incidencia no son comunes para los tres aislamientos, lo cual 
sugiere la existencia de posibles variaciones patogénicas o razas. Snyder (1933) mencionó la presencia de las razas 1 y 2 de $F$. oxysporum descritas en 1928 y 1933 respectivamente, mientras que Haglund and Kraft (1979) reportaron las razas 5 y 6 . Según Hagedorn (1984), cuatro genes han sido reconocidos como responsables de la resistencia a razas individuales de $F$. oxysporum f. sp. pisi. Por otra parte Checa (1993), utilizando líneas diferenciales, encontró que en aislamientos de F. oxysporum f. sp. pisi procedentes de los municipios de Pasto, Ipiales, Funes y Yacuanquer, en Nariño, existía variación entre los genes de virulencia, considerando la posible presencia de dos razas distintas a las reportadas anteriormente. No obstante, en la búsqueda de genotipos resistentes es posible evaluar en condiciones de invernadero y campo, su reacción a las razas prevalentes del patógeno en cada localidad.

Decoloración de raíz (DRAIZ). Para el aislamiento de Gualmatán los porcentajes de plantas con decoloración de raíz oscilaron entre 10,18 y $79,8 \%$ (Tabla 5). Si se compara estos valores con los observados en el porcentaje de amarillamiento que fueron de 10,18 a 57,1\%, se puede deducir que existieron plantas en algunos genotipos que sin presentar amarillamiento mostraron decoloración de raíz. El testigo ANDICON y las líneas UN7325, UN7321, UN7115, UN7103, UN7370-1, UN7364, ILS3597 e ILS3593 fueron altamente afectados por la decoloración vascular con porcentajes entre 79,8 y 52,49\%. Las líneas UN7328, UN7324, UN7232-1, UN7313 (y el testigo Andina sin inoculo), menos de $28,3 \%$ de

Tabla 4. Porcentaje de Amarillamiento 30 días después de inocular 18 líneas y dos testigos de arveja voluble (Pisum sativum L.) con tres aislamientos de Fusarium oxysporum f. sp. pisi (Datos trasformados arcoseno $\sqrt{\%}$ ).

\begin{tabular}{|c|c|c|c|c|c|c|c|c|}
\hline \multicolumn{3}{|c|}{ AISLAMIENTO GUALMATÁN } & \multicolumn{3}{|c|}{ AISLAMIENTO IPIALES } & \multicolumn{3}{|c|}{ AISLAMIENTO PUPIALES } \\
\hline UN7103 & 57,1 & a & UN7313 & 44,99 & a & ANDICON & 79,8 & a \\
\hline UN7143-2 & 55,38 & a & ILS3593 & 42,11 & $a b$ & UN7100 & 79,8 & a \\
\hline UN7100 & 55,38 & a & UN7336 & 42,11 & $a b$ & UN7143-1 & 71,62 & $\mathrm{ab}$ \\
\hline ILS3597 & 52,49 & $a b$ & UN7103 & 42,11 & $a b$ & ILS3621 & 67,06 & $a b c$ \\
\hline UN7364 & 46,78 & $a b$ & ANDICON & 41,98 & $\mathrm{ab}$ & UN7371-2 & 65,28 & $a b c$ \\
\hline UN7143-3 & 45 & $a b$ & UN7143-3 & 39,22 & $\mathrm{ab}$ & UN7324 & 65,28 & $a b c$ \\
\hline UN7324 & 45 & $\mathrm{ab}$ & UN7371-2 & 32,89 & $\mathrm{ab}$ & UN7103 & 57,1 & abcd \\
\hline UN7328 & 43,28 & $a b c$ & ILS3621 & 30,81 & $a b$ & UN7115 & 57,1 & abcd \\
\hline ILS3593 & 42,11 & $a b c$ & UN7100 & 29,99 & $a b$ & UN7325 & 45 & bcde \\
\hline UN7371-2 & 40,38 & $a b c$ & UN7143-1 & 29,99 & $a b$ & UN7143-2 & 45 & bcde \\
\hline ANDICON & 40,03 & $a b c$ & UN7328 & 28,28 & $a b$ & UN7313 & 45 & bcde \\
\hline UN7232-1 & 39,23 & $a b c$ & UN7324 & 26,56 & $a b$ & UN7232-1 & 45 & bcde \\
\hline UN7313 & 32,89 & $a b c$ & UN7325 & 26,56 & $a b$ & UN7336 & 45 & bcde \\
\hline UN7143-1 & 28,28 & $a b c$ & UN7364 & 18,37 & $a b$ & ILS3597 & 42,11 & bcdef \\
\hline UN7325 & 25,59 & $a b c$ & UN7370-1 & 14,99 & $a b$ & UN7328 & 34,61 & cdef \\
\hline UN7115 & 26,56 & $a b c$ & UN7232-1 & 10,18 & b & UN7143-3 & 28,28 & def \\
\hline UN7336 & 18,37 & bc & UN7143-2 & 10,18 & b & UN7370-1 & 20,09 & ef \\
\hline ILS3621 & 10,18 & $\mathrm{c}$ & UN7115 & 10,18 & $\mathrm{~b}$ & UN7364 & 10,18 & $f$ \\
\hline UN7370-1 & 10,18 & c & ILS3597 & 10,18 & b & ILS3593 & 10,18 & $f$ \\
\hline ANDISIN & 10,18 & c & ANDISIN & 10,18 & b & ANDISIN & 18,18 & $f$ \\
\hline Prom. Loc. & 36,22 & & & 27,09 & & & 46,58 & \\
\hline Comp. Tukey & 34,58 & & & & & & & \\
\hline
\end{tabular}

Valores con diferente letra son significativos al nivel del 5\%. Prueba de Tuckey. 
plantas afectadas, mostraron mayor resistencia con diferencias significativas respecto al testigo ANDICON (Andina con inoculación) y a la línea UN7325 que presentaron 79,8 y $67,71 \%$ de plantas con decoloración vascular respectivamente (Tabla 5).

Para el aislamiento de Ipiales, la decoloración vascular varió entre 10,18 y 69,89\% siendo mayor al rango de porcentaje de amarillamiento de plantas, que para este mismo aislamiento estuvo entre 10,18 y 44,99\% confirmando que existen plantas que sin mostrar el síntoma de amarillamiento presentan síntoma de enrojecimiento en su raíz. En este aislamiento, UN7100, UN7336, ILS3593 y UN7328 con promedios de 10,18\% mostraron reacción de resistencia, con diferencias al 0.05 de probabilidad en relación con UN7143-1;
ILS3621, UN7371-2, UN7371-1, UN71432, ANDICON (Andina con inoculación), ILS3597 y UN7103 que presentaron entre 69,8 y $42,11 \%$. (Tabla 5). En el aislamiento de Pupiales los promedios de genotipos evaluados variaron entre 10,18 y $71,61 \%$ (Tabla 5 ), Las líneas ILS3621, UN7325, UN7336, ILS3597, UN7328, constituyen el grupo de menor incidencia de decoloración de raíz con promedios de $10,18 \%$ presentando diferencias significativas respecto a UN7324, Andina con inoculo, UN72321, UN7364, UN73701, UN73712, UN7115 e ILS3597 que obtuvieron promedios entre 71,61 y $42,11 \%$, (Tabla 5).

En general, se pudo observar que en dos de los tres aislamientos los rangos para decoloración vascular de raíz fueron más amplios y con valores más altos que los de amarillamiento,

Tabla 5. Porcentaje de decoloración de raíz 30 días después de inocular 18 líneas y dos testigos de arveja voluble (Pisum sativum L.) con tres aislamientos de Fusarium oxysporum f. sp. pisi. (Datos trasformados arcoseno $\sqrt{\%}$ ).

\begin{tabular}{|c|c|c|c|c|c|c|c|c|}
\hline \multicolumn{3}{|c|}{ AISLAMIENTO GUALMATÁN } & \multicolumn{3}{|c|}{ AISLAMIENTO IPIALES } & \multicolumn{3}{|c|}{ AISLAMIENTO PUPIALES } \\
\hline ANDICON & 79,8 & $\mathrm{a}$ & UN7143-1 & 69,89 & a & UN7324 & 71,61 & a \\
\hline UN7325 & 61,71 & $a b$ & ILS3621 & 67,06 & a & ANDICON & 67,06 & $a b$ \\
\hline UN7115 & 57,09 & $a b c$ & UN7371-2 & 65,28 & $\mathrm{ab}$ & UN7232-1 & 65,28 & $\mathrm{ab}$ \\
\hline UN7103 & 57,09 & $a b c$ & UN7370-1 & 47,87 & $a b c$ & UN7364 & 62,39 & $a b$ \\
\hline UN7370-1 & 54,32 & $a b c$ & UN7143-2 & 44,99 & $a b c$ & UN7370-1 & 55,37 & $a b c$ \\
\hline UN7364 & 52,54 & $a b c$ & ANDICON & 44,99 & $a b c$ & UN7371-2 & 50,76 & $a b c$ \\
\hline ILS3597 & 52,49 & $a b c$ & ILS3597 & 42,11 & $\mathrm{abc}$ & UN7115 & 44,99 & $a b c$ \\
\hline ILS3593 & 52,49 & $a b c$ & UN7103 & 42,11 & $a b c$ & ILS3597 & 42,11 & $a b c$ \\
\hline UN7100 & 47,87 & $\mathrm{bc}$ & UN7115 & 34,61 & bcd & UN7143-1 & 39,22 & bcd \\
\hline UN7143-3 & 42,99 & bc & UN7324 & 32,89 & $\mathrm{~cd}$ & UN7143-2 & 39,22 & bcd \\
\hline UN7371-2 & 42,11 & bc & UN7313 & 32,89 & $\mathrm{~cd}$ & UN7143-3 & 28,28 & $\mathrm{~cd}$ \\
\hline UN7143-2 & 34,61 & bcd & UN7232-1 & 28,28 & $\mathrm{~cd}$ & UN7100 & 28,28 & $\mathrm{~cd}$ \\
\hline ILS3621 & 34,61 & bcd & UN7143-3 & 26,56 & $\mathrm{~cd}$ & UN7313 & 26,56 & $\mathrm{~cd}$ \\
\hline UN7143-1 & 34,61 & bcd & UN7325 & 26,56 & $\mathrm{~cd}$ & UN7103 & 26,56 & $\mathrm{~cd}$ \\
\hline UN7336 & 32,84 & bcd & UN7364 & 18,37 & $\mathrm{~cd}$ & ILS3621 & 10,18 & $d$ \\
\hline UN7328 & 28,28 & $\mathrm{~cd}$ & UN7100 & 10,18 & $d$ & UN7325 & 10,18 & d \\
\hline UN7324 & 26,56 & $\mathrm{~cd}$ & UN7336 & 10,18 & d & UN7336 & 10,18 & d \\
\hline UN7232-1 & 26,56 & $\mathrm{~cd}$ & UN7328 & 10,18 & d & UN7328 & 10,18 & d \\
\hline UN7313 & 26,56 & $\mathrm{~cd}$ & ILS3593 & 10,18 & d & ILS3593 & 10,18 & d \\
\hline ANDISIN & 10,18 & $d$ & ANDISIN & 10,18 & d & ANDISIN & 10,18 & d \\
\hline Prom. Loc. & 42,77 & & & 33,77 & & & 35,44 & \\
\hline Comp. Tukey & 30,95 & & & & & & & \\
\hline
\end{tabular}

Valores con diferente letra son significativos al nivel del 5\%. Prueba de Tuckey. 
mientras que para el aislamiento de Pupiales, la decoloración vascular fue ligeramente más baja. Lo anterior indica que es frecuente encontrar plantas con decoloración de raíz y sin amarillamiento y también aunque con menor frecuencia se pueda encontrar plantas con amarillamiento y sin decoloración vascular en su raíz, caso en el cual el amarillamiento no se puede atribuir al patógeno. Los resultados confirman los observaciones de Checa (1993) y Rodríguez (2006), quienes reportaron que en estados tempranos de la enfermedad, las plantas presentan una apariencia externa normal, observándose el mismo comportamiento en el sistema radical; sin embargo cuando la raíz se secciona longitudinalmente, esta puede tener un color que cambia de amarillo, a anaranjado o rojo oscuro en el tejido vascular, que se extiende hasta el epicótilo y la parte basal del tallo de la planta infectada.

En plantas con síntomas de decoloración vascular de raíz, pero sin amarillamiento es posible que el patógeno no haya logrado alcanzar a afectar con intensidad los haces vasculares del xilema y por lo tanto, la planta no tuvo dificultades para su nutrición hasta el momento de la evaluación. También es importante considerar que la reacción de resistencia puede darse después que el patógeno ingresa a la raíz, mediante el bloqueo de los conductos de las raíces afectadas y la producción abundante de nuevas raíces con la cual la planta no llega a presentar síntoma de amarillamiento. Según Charchar y Kraft (1989), las variedades resistentes al patógeno impiden el avance del hongo sellando los elementos del xilema por medio de geles o gomas. Beckam (1987), asegura que las defensas físicas tales como tilosas, geles y formación de gomas, restringen el movimiento de los patógenos. No obstante, se espera que una planta sea altamente resistente cuando no presente síntomas de amarillamiento ni decoloración de raíz. En un examen más cuidadoso, se observan hojas enroscadas con márgenes caídos (Arjona 1997).
Determinación del grado de resistencia en la escala 0 - 5 a partir de incidencia de amarillamiento y decoloración de raíz en invernadero.

Aislamiento de Gualmatán. Para el porcentaje de amarillamiento las líneas ILS3621 y UN7370-1 con calificación de cero se portaron como inmunes, mientras que UN7115 y UN7336 fueron resistentes, con calificación 1. Por otra parte, las líneas UN7143-1, UN72321 y UN7313 se mostraron como moderadamente resistentes. Los demás genotipos mostraron reacciones entre moderadamente susceptible y susceptibles, el testigo Andina fue moderadamente susceptible (Tabla 6).

Para porcentaje de decoloración de raíz o enrojecimiento, las líneas UN7232-1, UN7313 y UN7324 en Gualmatán, mostraron reacción resistente con calificación 1 , en tanto que ILS3621, UN7143-1, UN7143-2, UN7328 y UN7336 fueron moderadamente resistentes con calificación 2. Los demás genotipos se estuvieron entre moderadamente susceptibles y altamente susceptibles, la variedad Andina fue altamente susceptible. La coincidencia en la calificación de la reacción medida por porcentaje de amarillamiento frente a la medición hecha por decoloración de raíz escala 0 a 5 fue de solo $35 \%$.

Aislamiento de Ipiales: Las líneas ILS3597, UN7115, UN7143-2 y UN7232-1 no presentaron plantas con amarillamiento calificándose como inmunes (0), por su parte las líneas UN7324, UN7325 y UN7364 obtuvieron una reacción de resistencia y las líneas ILS3621, UN7100, UN7143-1, UN7143-3, UN7328, UN7370-1 y UN7371-2 alcanzaron una calificación de moderadamente resistentes. Los demás tratamientos oscilaron entre moderadamente susceptibles y altamente susceptibles. El testigo Andina fue moderadamente susceptible (Tabla 6). 
Tabla 6. Incidencia de amarillamiento y decoloración de raíz a la reacción de 18 líneas y dos testigos de arveja voluble (Pisum sativum L.) con tres aislamientos de Fusarium oxysporum f. sp. pisi bajo condiciones controladas.

\begin{tabular}{|c|c|c|c|c|c|c|}
\hline \multirow{2}{*}{ GENOT } & \multicolumn{2}{|c|}{ GUALMATÁN } & \multicolumn{2}{|c|}{ IPIALES } & \multicolumn{2}{|c|}{ PUPIALES } \\
\hline & $\begin{array}{l}\text { AMAR } \\
\text { GRADO }\end{array}$ & $\begin{array}{l}\text { ENROJ } \\
\text { GRADO }\end{array}$ & $\begin{array}{l}\text { AMAR } \\
\text { GRADO }\end{array}$ & $\begin{array}{l}\text { ENROJ } \\
\text { GRADO }\end{array}$ & $\begin{array}{l}\text { AMAR } \\
\text { GRADO }\end{array}$ & $\begin{array}{l}\text { ENROJ } \\
\text { GRADO }\end{array}$ \\
\hline Andicon & 3 & 5 & 3 & 4 & 5 & 5 \\
\hline Andisin & 0 & 0 & 0 & 0 & 0 & 0 \\
\hline ILS3593 & 3 & 4 & 3 & 0 & 0 & 0 \\
\hline ILS3597 & 4 & 4 & 0 & 3 & 3 & 3 \\
\hline ILS3621 & 0 & 2 & 2 & 5 & 5 & 0 \\
\hline UN7100 & 4 & 3 & 2 & 0 & 5 & 2 \\
\hline UN7103 & 4 & 4 & 3 & 3 & 4 & 1 \\
\hline UN7115 & 1 & 4 & 0 & 2 & 4 & 3 \\
\hline UN71431 & 2 & 2 & 2 & 5 & 5 & 2 \\
\hline UN71432 & 4 & 2 & 0 & 3 & 3 & 2 \\
\hline UN71433 & 3 & 3 & 2 & 1 & 2 & 2 \\
\hline UN72321 & 2 & 1 & 0 & 2 & 3 & 5 \\
\hline UN7313 & 2 & 1 & 3 & 2 & 3 & 1 \\
\hline UN7324 & 3 & 1 & 1 & 2 & 5 & 5 \\
\hline UN7325 & 3 & 4 & 1 & 1 & 3 & 0 \\
\hline UN7328 & 4 & 2 & 2 & 0 & 2 & 0 \\
\hline UN7336 & 1 & 2 & 3 & 0 & 3 & 0 \\
\hline UN7364 & 4 & 4 & 1 & 1 & 0 & 5 \\
\hline UN73701 & 0 & 4 & 2 & 3 & 2 & 4 \\
\hline UN73712 & 3 & 3 & 2 & 5 & 5 & 3 \\
\hline
\end{tabular}

Escala de evaluación 0 = Inmune; 1 = Resistente; 2 = Moderadamente Resistente; $3=$ Moderadamente Susceptible; $4=$ Susceptible y $5=$ Altamente Susceptible.

Para enrojecimiento de raíz las líneas UN7100, UN7328 y UN7336 se mostraron inmunes (0), mientras que UN7325, UN7364 y UN7143-3 fueron resistentes y las líneas UN7115, UN73321, UN7313 y UN7324 fueron moderadamente resistentes, las demás se ubicaron entre moderadamente susceptibles y altamente susceptibles, el testigo Andina fue susceptible. La coincidencia en las calificaciones de amarillamiento y enrojecimiento en la escala de 0 a 5 fue de solo un $20 \%$.

Aislamiento de Pupiales: Con respecto al porcentaje de plantas con amarillamiento las líneas ILS3593; UN7364 tuvieron una calificación (0) que las ubica como inmunes; las líneas UN7143-3, UN7328 y UN73701 tuvieron una reacción moderadamente resistente; las demás líneas estuvieron entre moderadamente susceptibles y altamente susceptibles, el testigo Andina fue altamente susceptible (Tabla 6).

Con relación al porcentaje de enrojecimiento las líneas ILS3593, ILS3521, UN7325, UN7328 y UN7336 fueron calificadas como inmunes en la escala de 0 a 5 por no presentar plantas con decoloración de raíz; las líneas UN7103, UN7313 tuvieron una reacción de resistencia con calificación 1; las líneas UN7100, UN7143-1, UN7143-2 y UN7143-3 alcanzaron una reacción de moderadamente resistente calificación 2. Las demás líneas mostraron una reacción entre moderadamente susceptibles y altamente susceptibles. El testigo Andina se mostró altamente susceptible.

En Pupiales la coincidencia en la calificación 
de la reacción obtenida por las líneas para porcentaje de amarillamiento y porcentaje de enrojecimiento de raíz observada en invernadero y tomada en la escala de 0 a 5 fue del $30 \%$.

El bajo porcentaje de coincidencia en las calificaciones obtenidas por las líneas en el porcentaje de amarillamiento y porcentaje de decoloración vascular, sugiere que es necesario tener en cuenta simultáneamente las dos variables para identificar las líneas de mejor respuesta. En consecuencia para Gualmatán se destacaron los genotipos ILS3621, UN7143-1, UN7232-1, UN7313 y UN7336 por presentar valores entre 0 y 2 mostrandoal menos moderada resistencia. En Ipiales las líneas UN7325 y UN7364 fueron resistentes con calificación 1 para amarillamiento y decoloración vascular y los genotipos UN7100, UN7115, UN7328, UN7143-3, UN7232-1 y UN7324 presentaron calificación 2 para alguna de las dos variables amarillamiento y decoloración vascular, que los ubicaron como moderadamente resistentes (Tabla 6). Finalmente, en Pupiales sobresale la línea ILS3593 con calificación 0 (Inmune), para los dos conceptos (amarillamiento y decoloración vascular) y las líneas UN71433 y UN7328 con una reacción de moderada resistencia al presentar calificación 2 en alguno de los dos conceptos.

Ninguna de las líneas mostró resistencia o moderada resistencia para los tres aislamientos en conjunto. De las 18 líneas estudiadas UN7143-3 fue moderadamente resistente a los aislamientos de Ipiales y Pupiales y moderadamente susceptible para el aislamiento de Gualmatán, de igual manera la línea UN7232-1, presentó moderada resistencia para los aislamientos de Gualmatán e Ipiales, y la línea UN7328 presentó moderada resistencia en los aislamientos de Ipiales y Pupiales (Tabla 6).

En general, la baja coincidencia en las evaluaciones de los genotipos en la escala
0-5, entre porcentaje de amarillamiento de plantas y porcentaje de decoloración vascular de raíz, sugiere como se dijo antes que plantas sin amarillamiento pueden presentar enrojecimiento en la raíz. Es posible que hasta los 30 días después de la inoculación, en algunas líneas exista penetración del patógeno pero no invasión de la raíz, sin embargo estas plantas hacen parte de las afectadas por el patógeno de acuerdo con la metodología de evaluación propuesta. En consecuencia, la susceptibilidad al patógeno requiere que los dos síntomas estén presentes en la planta. Al respecto Stulher y Heidi (1989), evaluaron la reacción de genotipos a razas de $F$. oxysporum usando el índice de marchitez de Ebbels y encontraron dificultad en distinguir la reacción susceptible de la resistente. Por lo tanto, sugirieron evaluar algunas características adicionales o alternativas como decoloración vascular, colonias de Fusarium obtenidas de los nudos 4 y 6 , número de nudos por planta y longitud de las plantas. En la presente investigación, la longitud de las plantas hasta los 30 días no logró diferenciar la reacción susceptible de la resistente.

En futuras investigaciones, la evaluación por reacción a $F$. oxysporum no solo debe contemplar la incidencia, sino también una escala de severidad que permita medir el avance del patógeno en la invasión de la raíz de la planta afectada. Es importante que una vez detectada la presencia del patógeno en la raíz, se evalúen fuentes de resistencia parcial, con base en el grado de afección. Grünwald et al. (2003), utilizaron una escala de severidad de 0 a 5 para medir en arveja la resistencia parcial a la pudrición de raíz (F. solani). Finalmente, el resultado observado tanto en la evaluación estadística de porcentaje de amarillamiento y decoloración vascular, como en la medición de reacción en la escala de 0-5, sugiere variabilidad interna en las líneas evaluadas, lo cual es probable, si se tiene en cuenta que las mismas son líneas recombinantes que fueron uniformizadas a partir de la generación 
F3 y hasta F6 mediante selección masal por componentes de rendimiento y en ningún caso, se uniformizaron por su respuesta a F. oxysporum. En consecuencia es posible aprovechar la variación observada dentro de ellas, para realizar selecciones individuales que generen nuevas líneas con una resistencia uniforme.

\section{CONCLUSIONES}

Los aislamientos del hongo Fusarium oxysporum f. sp. pisi procedentes de Gualmatán, Ipiales y Pupiales en Nariño no afectaron la altura de planta de los 18 genotipos de arveja evaluados hasta los 30 días después de la inoculación del patógeno.

La coincidencia entre el porcentaje de amarillamiento y decoloración de raíz fue inferior al 35\% indicando que los dos síntomas deben ser evaluados en conjunto para determinar la susceptibilidad de la planta.

Existió variabilidad genética en la reacción a Fusarium oxysporum f. sp. pisi dentro de las líneas evaluadas al presentar plantas susceptibles y resistentes.

Los genotipos mostraron un comportamiento diferencial a través de los aislamientos de Fusarium oxysporum. Para el aislamiento Gualmatán, cinco genotipos fueron moderadamente resistentes; para el aislamiento Ipiales dos genotipos fueron resistentes y seis moderadamente resistentes y para el aislamiento Pupiales una línea fue inmune y dos moderadamente resistentes.

Ninguna de las líneas fue resistente o moderadamente resistente a los tres aislamiento de Fusarium oxysporum. Se destacaron por presentar moderada resistencia para dos de los tres aislamientos las líneas UN7232-1 UN71433 y UN7328.

\section{AGRADECIMIENTOS}

Los autores manifiestan su agradecimiento a Colciencias por la Financiación del proyecto, a la Universidad de Nariño y al Servicio Nacional de Aprendizaje Sena por su apoyo en la logística de los ensayos de invernadero, a Marcela Riascos y Jaime López por su apoyo en el montaje y manejo de los ensayos de invernadero.

\section{REFERENCIAS}

Andrés J., Collar J., Iglesias C. y Tello J. 1999. Evaluación de la resistencia de variedades comerciales de clavel de patotipos de Fusarium oxysporum f. Sp. Dianthi detectados en Galicia. Boletín de sanidad vegetal. Plagas, 25(2), 209-220.

Avendaño, C., Arbeláez, G. y Rondón, G. 2006. Control biológico del marchitamiento vascularcausado por Fusarium oxysporum f. sp. phaseoli en fríjol Phaseolus vulgaris L., mediante la acción combinada de Entrophospora colombiana, Trichoderma sp. y Pseudomonas fluorescens. Revista Agronomía Colombiana. Universidad Nacional de Colombia. Vol. $24 \mathrm{~N}^{\circ} 1$. Bogotá. 5p, 24(1), 62-67.

Beckman, C. 1987. The nature of wilt disease of the plants APS. The American Phytopathological Society ST. APS press. St Paul, MN, U.S.A. 175p.

Benavides, M. y Muñoz, H. 1998. Efectos de la inoculación de un Trichoderma sp. y Pseudomonas fluorescens a fuente comercial de micorigenizados sobre la incidencia del amarillamiento Fusarium oxysporum f. sp. pisi y los componentes productivos de la arveja (Pisum sativum L.) Variedad santa Isabel en el municipio de Yacuanquer. Trabajo de Grado (Ingeniería Agronómica). Universidad de 
Nariño. Facultad de Ciencias Agrícolas. Pasto. 110p.

Buitrago, J., Duarte, C. y Sarmiento, A. 2006. El cultivo de la arveja en Colombia. Primera Edición. FENALCE, Fondo Nacional de Leguminosas. Bogotá, D. C., Colombia: s.n. 83p.

Castellanos, G., Jara, C. y Mosquera, G. 2011. Guías prácticas de laboratorio para el manejo de patógenos del frijol. Centro Internacional de Agricultura Tropical. Cali, Colombia. 4-2 a 4-32p.

Charchar, M. y Kraft, M. 1989. Response of near- isogenic pea cultivars to infection by Fusarium oxysporum f. sp. pisi, races 1 and 5. Canadian Journal of Plant Science 69, 1335-1346.

Checa, O. 1993. Determinación de razas del hongo Fusarium oxysporum f. sp. pisi y su efecto en materiales de arveja en el departamento de Nariño. Tesis para optar al título de Magister en producción vegetal con énfasis en fitomejoramiento. Universidad Nacional de Colombia, Sede Palmira. Colombia. 110p.

ICA, IGAC, 1985. Zonas agroecológicas de Colombia.

Forero, A. y Ligarreto, G. 2009. Evaluación de dos sistemas de tutorado para el cultivo de arveja (Pisum savitum L.) en condiciones de la Sabana de Bogotá. Revista de ciencias Hortícolas. 3(1): 81 - 94.

Grünwald, N., Coffman, V. and Kraft, J. 2003. Sources of partial resistance to Fusarium root rot in the Pisum core collection. Plant Dis. 87:1197-1200.

Hagerdorn, D. 1984. Compendium of the pea disease. The American Phytopathological Society. Minesota. 57p.
Haglund, W. y Kraft, J. 1979. Fusarium oxysporum f. sp pisi race 6 ocurrence and distribution. Phytopathology 60: 818 $-820$

Kraft, J. M. y Haglund, W. 1978. A reappraisal of the race classification of Fusarium oxysporum f. sp. pisi. Phytopathology (68): $273-275$.

Kraft, J. y Pfleger, F. 2000. Compendium of Pea Diseases and Pests. Second ed. The American Phytopathological Society. St. Paul, MN.7p

Leslie, J. and Summerell, B. 2006. The Fusarium laboratory manual (Vol. 2, No. 10). Ames, IA, USA: Blackwell Pub.387p.

Madriz, K. 2002. Mecanismos de defensa en las interacciones planta - patógeno. Manejo Integrado de Plagas (Costa Rica). (63): 22 -32 .

Martínez, M., Hernández, S., Padilla, J. y Mayec, N. 2004. Diversidad Patogénica y Genética de Aislamientos de Fusarium de Aguascalientes, México. Revista Mexicana de Fitopatología, vol. 22, núm. 3, diciembre, 2004, pp. 321-327

Muñoz, M. 2012. Interacción genotipo ambiente de 20 líneas de arveja arbustiva (Pisum sativum L.) para cinco municipios de la zona del sur del departamento de Nariño. Tesis de maestría. Pasto, Colombia. Facultad de Ciencias Agrícolas. Universidad de Nariño. 131p.

Ormeño, E y Zuñiga, D. 1999. Optimización del tiempo de esterilización de soportes basados en suelo y compost en la producción de inoculantes para leguminosas. Revista Peruana de Biología $6(2): 4$ 
Prieto, G. 2008. Pautas para el manejo del cultivo de Arveja. INTA. En:http://inta. gob.ar/documentos/pautas-para-elmanejo-del-cultivo-de arveja/at_multi_ download/file/Pautas-para-el-manejodel-cultivo-de-arveja-final.pdf. Consulta: Marzo 2012.

Quistial, J. y Chávez, D. 2009. Evaluación agronómica de 20 líneas de arveja voluble (Pisum sativum L.) en cinco municipios de Nariño. Tesis de grado Ingeniero Agrónomo. Facultad de Ciencias Agrícolas Universidad de Nariño. Pasto, Nariño. Colombia. 120 p.

Rodríguez, M. 2006. Determinación de razas fisiológicas de fusarium oxysporum $\mathrm{f}$. $\mathrm{p}$. pisi en las principales zonas productoras de arveja de Cundinamarca y Boyacá. Trabajo presentado como requisito parcial para optar al título de Magister en Ciencias énfasis Fitopatología. Facultad de Agronomía. Universidad Nacional de Colombia, Bogotá. 88p.
Sañudo, B., Checa, O. y Arteaga, G. 1999. Manejo agronómico de leguminosas en zonas cerealistas: La arveja. Fenalce, Universidad de Nariño, Profiza, Corpoica y Corpocebada. Produmedios. Pp 49-65, pág. 98.

Seifert, K. 1996. FUSKEY (Fusarium interactive key). Her majesty the queen in right of Canada, agriculture and angry-food Canada. 65p.

Stulher, I. y Heidi, J. 1989. Evaluation of alternative assessement of resistance in test of pea with Fusarium oxysporum f. sp. pisi. Research Report Pisum Newsletter 4 (21): 346-450

Tamayo, P. 2000. Enfermedades del cultivo de la arveja en Colombia: guía de reconocimiento y control. Boletín técnico. Corporación Colombiana de Investigación Agropecuaria-Corpoica. Regional 4. Rionegro, Antioquia. Colombia. 52p. 